\title{
A System of Heterogenesis: Deleuze on Plurality
}

\author{
Martijn Boven
}

In almost all of his early works, Gilles Deleuze is concerned with one and the same problem: the problem of genesis. In response to this problem, Deleuze argues for a system of heterogenesis. As is well-known, his position can be characterized as follows: identity is a particular case of difference, just like equality is a particular case of inequality and rest is a particular case of motion. In other words, identity is produced by difference and not the other way around. "I believe in philosophy as system," Deleuze states. "For me, the system must not only be in perpetual heterogeneity, it must be a heterogenesis-something which, it seems to me, has never been attempted." In other words, Deleuze defines plurality as a system of heterogenesis that organizes itself on the basis of the pure differences by which it is constituted. These pure differences produce the states of affairs in which they are embodied and simultaneously subsist within these states of affairs, without losing their power to produce new states of affairs. In Deleuze's view, it would be a mistake to define plurality in terms of diversity. Diversity is given, but Deleuze is interested in the difference of intensity "by which the given is given, that by which the given is given as diverse." 2 This difference of intensity is the productive condition of diversity, rather than diversity itself.

Deleuze rejects the traditional metaphysical opposition between the One and the Multiple. He argues that the organizing principle that gives structure and unity to plurality does not come from outside. Pluralities organize themselves, without any help from a transcendent cause that precedes them. Put differently, pluralities operate on the basis of an immanent cause. To conceptualize plurality, Deleuze borrows the term "multiplicity" from Henri Bergson's reading of the mathematician Bernhard Riemann. In his Bergsonism, Deleuze shows that "it is not a question of opposing the Multiple to the One but on the contrary of distinguishing two types of multiplicity." 3 In Difference and Repetition,

1 Gilles Deleuze, "Letter Preface," in Variations: The Philosophy of Gilles Deleuze, ed. Jean-Clet Martin, (Edinburgh: Edinburgh University Press, 2010), 8.

2 Gilles Deleuze, Difference and Repetition, trans. Paul Patton (London: Athlone Press, 2001), 222. Here and in other cases the translation is modified according to the American spelling.

3 Gilles Deleuze. Bergsonism, trans. Hugh Tomlinson and Barbara Habberjam (New York: Zone, 1991), 39. 
Deleuze redefines these two types of multiplicity in terms of virtual Ideas that are differentiated in themselves and intensive dramas that are implicated in each other. Plurality must be conceived as the interplay of these two multiplicities in which the diversity of actual concepts is produced. Hence, Deleuze's system of heterogenesis operates on three levels: (1) the differential multiplicity of virtual Ideas; (2) the implied multiplicity of intensive dramas; (3) the extensive and qualitative diversity of actual concepts.

The three central notions that I just introduced-Ideas, concepts and drama's-are derived from Difference and Repetition. As Deleuze writes, "[w]e distinguish Ideas, concepts and dramas, the role of dramas is to specify concepts by incarnating the differential relations and singularities of an Idea." 4 These three notions can be enriched, by combining them with Deleuze's famous distinction between the virtual Idea and the actual concept. To bridge the gap between the virtual and the actual, Deleuze introduces a third notion: intensive dramas. I will argue that virtual Ideas and intensive dramas correspond to the two multiplicities discussed above. The actual concepts, on the other hand, correspond to the diversity that is produced by these two multiplicities. As I hope to show, the relation between these three notions can be explained in terms of the logic of expression that Deleuze develops in Expressionism in Philosophy: Spinoza. ${ }^{5}$ In this way, I hope to gain clarity without losing nuance. The rather technical and abstract language of expression will become more concrete and understandable when it is understood in terms of intensive dramas, virtual Ideas and actual concepts. At the same time, the three levels of expression make it possible to show how these notions are interlinked. Accordingly, this article is divided in four parts. In the first part, I will explain how Deleuze takes up Kant's discovery of the principle of difference as a reaction against the model of representation. The second part focuses on the two multiplicities (virtual Ideas and intensive dramas) that produce diversity (actual concepts). In the third part, these two multiplicities are linked to each other with the help of the logic of expression that Deleuze derives from Spinoza. The fourth, concluding part will connect the logic of expression to the complex dynamic of difference and repetition.

4 Deleuze, Difference and Repetition, 218 (my emphasis).

5 Gilles Deleuze, Expressionism in Philosophy: Spinoza, trans. Martin Joughin (New York: Zone, 1990). 
In Difference and Repetition, Deleuze takes issue with the dominant tendency of Western philosophy to fall back on a model of representation that operates on the opposition between the One and the Multiple. According to Deleuze, this model of representation cannot account for real genesis because it subordinates difference-which includes the new-to a predetermined identity. This implies that representation distorts both difference (which makes genesis possible) and repetition (the fact that genesis takes place over and over again). Representation thus starts from two basic errors. The first error emerges because difference "is represented in the identical concept, and thereby reduced to a merely conceptual difference." ${ }^{6}$ It is only because representation already thinks to know what, for instance, a horse is that it can subdivide horses in different species. The difference between these species is internal to the concept of a horse in general (the genus). The second error is the result of representing repetition as a perfect resemblance, as "a difference without concept, but always with the presupposition of an identical concept."7 Take for instance, the difference between two grains of dust. From the perspective of representation, these differences are not determined by the grains themselves, but only come to light externally, in an already established spatial order. In this spatial order it is clear what a "grain of dust in general" is supposed to be. For that reason, the grains of dust are considered to be repetitions of the same. The difference between them is "a difference without concept" and therefore it is irrelevant. The model of representation results in a classification system that distributes being over all the existing entities (common sense) and orders them in a certain hierarchy (good sense). Therefore, from the perspective of representation, plurality is defined as a sedentary distribution that creates fixed boundaries in which everything has its place. An example of such a distribution would be a classroom in which each of the schoolchildren has his or her territory (the desk designated to them, their own little space on the bookshelf, etc.) "A distribution of this type," Deleuze writes, "proceeds by fixed and proportional determinations which may be assimilated to 'properties' or limited territories within representation." The problem with this type of distribution is, however, that it cannot explain how the distribution itself came into being. For that reason, Deleuze proposes a different conception of

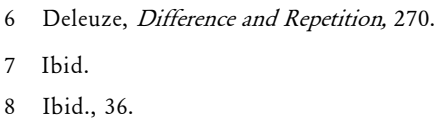


plurality in which being is not understood in terms of a sedentary distribution but in terms of a nomadic one.

Deleuze challenges the double reduction that underlies representation: the reduction of difference to an internal, but purely conceptual difference; the reduction of repetition to a non-conceptual, but purely external difference. As an alternative, Deleuze argues for a pure difference that is both non-conceptual and internal and for a repetition that no longer repeats the same, but that must be conceived as a repetition of pure differences. This amounts to a plurality that is defined as a nomadic distribution of being. This distribution operates in an open space without fixed boundaries in which everything is in flux. For instance, a wasteland in which playing children distribute themselves according to their own possibilities and preferences. Such a nomadic distribution is not determined by fixed claims of ownership, but rather by who is there and who is not (the presence of a friend leads to a different distribution than the presence of a bully, etc.). As Deleuze indicates, "there is no longer a division of that which is distributed but rather a division among those who distribute themselves in an open space-a space which is unlimited, or at least without precise limits." 9 For Deleuze, this nomadic distribution implies a different conception of plurality that is based on a new understanding of difference and repetition. It is the internal difference of intensity that creates the distribution (to stand up to the bully, to fight with the friend) and repeats itself in constantly differing variations (to withdraw at the last moment, to abandon the friend). According to Deleuze, this nomadic distribution opens the way to an ontology in which difference is no longer subordinated to identity. To move from a sedentary distribution to a nomadic one, Deleuze needs a method of genesis. According to him, it was Kant who came closest to such a method of genesis. Kant initiated a series of reversals that ultimately lead to the discovery of the principle of difference. ${ }^{10}$ However, Kant did not pursue this discovery. Instead of developing a method of genesis that can account for real experience, Kant reverted to a method of conditioning that can only account for possible experience. Deleuze goes back to the Kantian discovery to develop his system of heterogenesis.

According to Deleuze, the principle of difference emerged in the Kantian critique on the cogito ergo sum (I think therefore I am) argument. This argument introduced two logical values: a determination (I think) which specifies

9 Ibid.

10 Cf. Gilles Deleuze, “On Four Poetic Formula’s that Might Summarize the Kantian Philosophy,” in Essays Critical and Clinical, trans. Daniel W. Smith and Michael A. Greco (Minneapolis: University of Minnesota Press, 1997). 
an as yet undetermined existence ( $\mathrm{I} \mathrm{am}$ ) as a res cogitans (thinking being). In other words, for Descartes, the determinate self "I think" not only implies a previously undetermined self "I am," but also tells us how this "I am" is determined by the "I think." Kant disagrees. In his view, it still remains to be seen how the undetermined self (being) is specified by the determining self (thinking). For that reason, he introduces a third value: the determinable self (the passive self, understood as the receptivity of intuition). It is only because a determinable self is presupposed that the undetermined self $\mathrm{can}$ be specified as a determining self. According to Deleuze, Kant's introduction of this third value-the determinable-amounts to the discovery of difference. Deleuze defines it as "the form in which the undetermined is determinable (by the determination)." "However, Deleuze is quick to point out that Kant failed to draw out the implications of his discovery. In Kant's work "synthesis is understood as active and as giving rise to a new form of identity in the I [the determining self], while passivity is understood as simple receptivity without synthesis [the determinable self]." 12 For Kant, the introduction of the determinable only provided the conditions of possible experience. Therefore, Kant never reached an internal method of genesis but got stuck in an external method of conditioning. Deleuze, however, tries to develop a method of genesis that brings to light the conditions of real experience. To make this happen, he substitutes Kant's "receptivity without synthesis" for his own conception of a passive self. In his view, this passive self breaks down in a series of larval subjects that bring about a series of passive syntheses, preceding the active synthesis of the I. ${ }^{13}$

To understand Deleuze's position, it will be helpful to have a closer look at Kant's discovery of the individuating difference. Deleuze describes it as follows:

It amounts to the discovery of Difference-no longer in the form of an empirical difference between two determinations, but in the form of a transcendental Difference between the Determination as such and what it determines; no longer in the form of an external difference which

13 At this point, I will not be able to develop Deleuze's conception of the self. Strictly speaking the ultimate condition of real experience is what Deleuze calls the "pure and empty form of time." He states that "my undetermined existence can be determined only within time as the existence of a phenomenon, of a passive, receptive phenomenal subject appearing within time" (Deleuze, Difference, 86). Here I will only focus on the virtual Idea and leave the complex issue of this "pure and empty form of time" aside. 
separates, but in the form of an internal Difference which establishes an a priori relation between thought and being. ${ }^{14}$

This indicates that Deleuze's conception of difference can be understood with the help of the three logical values that Kant distinguished: the undetermined, the determined and the determinable. According to Deleuze, the determinable-as transcendental difference-is the condition under which the undetermined can get a certain determination. It should not be understood as an empirical difference between two things that are already determined. On the contrary, the determinable is a transcendental and intrinsic difference, a difference that precedes identity and cannot be subordinated to it. As such it creates the difference between the undetermined ("I am" or being) and the determination ("I think" or thinking). The determinable "distinguishes itselfand yet that from which it distinguishes itself does not distinguish itself from it." 15 To explain this rather cryptic formula, Deleuze introduces the example of lightning. "Lightning, for example, distinguishes itself from the black sky but must also trail it behind, as though it were distinguishing itself from that which does not distinguish itself from it." 16 In this example difference should not be understood as a difference between the lightning bolt (the figure) and the black sky (the background). Rather, both the lightning bolt and the black sky must be considered as pure variations that have no fixed identity but are in a constant process of becoming. In other words, difference must not be understood in terms of " $\mathrm{x}$ is not $\mathrm{y}$ " (the lightning bolt is not the dark sky) or "x differs from y" (the lightning bolt differs from the black sky), but rather as "the undetermined being of $\mathrm{x}$ differs from the determination of $\mathrm{x}$. " This simply means that the individuating difference is internal to the lightning bolt. The individuating difference that constitutes the lightning bolt can be understood as a positively and a negatively charged electron that enter in a differential relation. This relation initiates a process of constant variation in which the previously undetermined electron becomes determined as electricity. The differential relation defines this process. The lightning bolt is the visible form in which this process manifests itself. It comes into being because the tension of electricity is building up to a climax. To get a more secure grip on this process, Deleuze introduces two important notions: the virtual Idea and the actual concept. 
To explain the difference between the virtual and the actual, it might be helpful to quote Deleuze's comments on the Nietzschean distinction between the creation of new values and the recognition of established values. According to Deleuze, this distinction must not be understood "in a historically relative manner, as though the established values were new in their time and the new values simply needed time to become established." 17 To his mind, new values will never become established values. The new and the established differ in nature. As Deleuze explains, the "new, with its power of beginning and beginning again, remains forever new, just as the established was always established from the outset, even if a certain amount of empirical time was necessary for this to be recognized."18 Nietzsche's distinction between the new and the established corresponds to Deleuze's distinction between the virtual Idea and the actual concept. The new corresponds to a virtual and transcendental domain of Ideas that will always remain unknown and cannot be identified. The established corresponds to an actual and empirical domain of concepts that can be known and identified. It is the virtual process of constant variation that establishes something, but this "something" is not the virtual process itself, it is the actual thing that springs from it. In other words, it is the virtual Idea that initiates the power of beginning and beginning again, but it is the actual concept that is originated in this process. Moreover, for Deleuze, it is of the utmost importance that the virtual Idea is not confused with a mere possibility. "The possible," he writes, "is opposed to the real; the process undergone by the possible is therefore a 'realization'. By contrast, the virtual is not opposed to the real; it possesses a full reality by itself. The process it undergoes is that of actualization." 19 The possible-but-not-real can in principle be expressed by the real, even if it is not realized at the moment. The virtual-but-real Idea can only be expressed in the actual concept by changing its nature, i.e. in a displaced and disguised form. When the virtual Idea is actualized it does not become more real, but it gets a particular modification that reduces the excess of its virtual determination.

In order to understand the process of beginning and beginning again that is initiated by the individuating difference, the domain of the virtual and the domain of the actual need to be complemented with a third domain that relates them to each other. This is the domain of intensive dramas. According to Deleuze, the virtual Idea and the intensive drama are "two corresponding

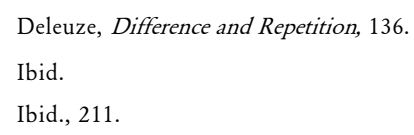


figures of difference." 20 The virtual Idea encloses the difference by specifying its differential relation, whereas the intensive drama makes sure that the difference individuates itself by a process of intensification, which will gradually lead to its actualization. Deleuze defines both the virtual Idea and the intensive drama as multiplicities. In Deleuze's view, a "multiplicity" does not "designate a combination of the many and the one, but rather an organization belonging to the many as such, which has no need whatsoever of unity in order to form a system." 21 This self-organizing multiplicity can be characterized in terms of the three Kantian notions that were explained at the beginning of this section: the undetermined, the determination and the determinable.

\section{Two Multiplicities: Virtual Ideas and Intensive Dramas}

As I indicated earlier, Deleuze's conception of plurality implies a nomadic distribution. This nomadic distribution is made possible by an interplay of two types of multiplicity: a pre-individual differential multiplicity that is organized by the virtual Idea and an individualizing implicated multiplicity that is animated by the intensive drama. The relation between the two multiplicities can be explained with the help of the example of the color green that Deleuze uses in The Fold. As Deleuze indicates, the color green is the result of a series of unconscious perceptions of infinitely small elements of yellow and blue. The Idea green is produced whenever these invisible, but distinct elements of yellow and blue enter into a relation with each other. Deleuze calls this a "differential relation," i.e. a relation between two infinitely small virtual elements that are real, but not actual (Deleuze calls these virtual elements differentials)." 22 Whenever a virtual series of unconscious perceptions of blue enter into a relation with a virtual series of unconscious perceptions of yellow, this will result in the color green. According to Deleuze, "yellow and blue can surely be perceived, but if their perception vanishes by dint of progressive

20 Ibid., 244.

21 Ibid., 182.

22 In an attempt to find a language in which virtual multiplicities can be described, Deleuze turns to the calculus as it was developed by Leibniz. In the language of the calculus, the undetermined must be understood as infinitely small elements that are called differentials (dx, dy); the determinable, as condition, is defined as the differential relation $(\mathrm{dx} / \mathrm{dy})$; the determination can be characterized in terms of a Taylor series (potential values of $\mathrm{dx} / \mathrm{dy}$ ). For a rigid, but also quite dense and inaccessible account of Deleuze's relation to mathematics, cf. Simon Duffy, The Logic of Expression: Quality, Quantity, and Intensity in Spinoza, Hegel, and Deleuze (Aldershot: Ashgate, 2006). 
diminution, they enter into a differential relation that determinates green."23 In this process green is produced. Green comes into being at the singular point at which yellow and blue enter into a differential relation; it will cease to exist when this differential relation breaks down. This process can be split up into two moments.

The first moment is related to the virtual Idea green and the differential multiplicity that corresponds to it. As became clear earlier, this multiplicity can be characterized in terms of the undetermined, the determination and the determinable. What would this imply for the color green? First, the undetermined can be defined as the infinite virtual totality of potential unconscious perceptions of yellow and blue. Taken by themselves, these unconscious perceptions are undetermined by green. Neither yellow nor blue is determined by green, but green emerges whenever they enter into a certain relation to each other. Second, Deleuze defines the determinable as a differential relation "in the reciprocal synthesis of the Idea." 24 The virtual Idea implies a synthesis between two undetermined virtual elements. This synthesis is reciprocal, because these virtual elements only exist as such in the synthesis between them. They determine each other or, rather, they are determined by the differential relation between them. The differential relation does not determine actual instances of yellow and blue as green. It only specifies the condition under which virtual differentials of yellow and blue will turn into green, i.e. in their reciprocal synthesis. As Deleuze indicates, this is no longer a condition of possible experience (as in Kant), but a condition of real experience. Third, the determination can be defined as the excess of differences that is determinable by the Idea green. In other words, all the potential instances in which the relation between two tiny perceptions (blue and yellow) will result in green. Although this totality is infinite and unknowable, it is restricted by two limits: the moment that yellow vanishes (green becomes blue) and the moment that blue vanishes (green becomes yellow). These limits define the range of potential variations within the differential relation of green. Green becomes darker when blue is more intense than yellow; it becomes lighter when it is the other way around. In other words, green is an individuating difference that has no fixed identity but that is defined by its limits. Because of these limits the infinite totality of potential variations is virtually determinable. That is why Deleuze can say that this virtual totality is real and not just possible. 88.

24 Deleuze, Difference and Repetition, 244. 
The differential multiplicity of the virtual Idea green:

- The virtually undetermined. The infinite totality of the differentials "yellow" and "blue" that can become virtually determined as green through a reciprocal relation (yellow and blue as differentials)

- The virtually determinable. The conditions under which something is virtually determinable as green: the differential relation between yellow and blue (yellow/blue)

- The virtual determination. The excess of the virtual Idea green, i.e. the infinite totality of potential instances virtually determined as green (the range of variations implied by yellow/blue)

When one asks, "how can something that is virtually undetermined by the Idea green, become virtually determined by it?" Deleuze's answer will be, "when virtual perceptions of yellow and virtual perceptions of blue enter into a differential relation." This differential relation defines the limits of the action of green, its range of existence. When either yellow or blue becomes too dominant and the differential relation between them breaks down, the Idea green will no longer apply. This implies that the differential relation, as the condition of green, is not defined by human reflection after the fact, but is part of the internal organization of a differential multiplicity.

The second moment within the process of becoming green is related to the actualization of the Idea green. This individuation is the result of an intensive drama that expresses the differential relation characterizing green. To the intensive drama corresponds an implicated multiplicity that can also be described in terms of the undetermined, the determinable and the determination. First, the actual undetermined can be defined as the excess of the virtual Idea. Although this excess is virtually determined, it has not yet become actually determined. It defines the infinite totality of potential instances of green, but it does not say anything about the actualization of these instances. Second, Deleuze defines the actual determinable as a relation of intensity "in the asymmetrical synthesis of the sensible. ${ }^{.25}$ The intensive drama creates a synthesis between the virtual Idea, as defined by its differential relation, and the actual circumstances in which this idea is incarnated. This synthesis is asymmetrical and not reciprocal. This is because the virtual Idea underlies the actual circumstances in which it is incarnated, without being determined by them. In other words, the synthesis only goes one way-from virtual to actual-and therefore

25 Ibid. 
it is not reciprocal but asymmetrical. Third, the actual determination, as the result of the asymmetrical synthesis, amounts to a reduction of difference. We saw that the virtual Idea remains infinitely differentiated, even though this infinity is fixed between two limits (defined by the differential relation). However, at the moment the virtual Idea becomes incarnated in actual circumstances, its infinite differentiation-or excess-is reduced to only a small number of its potential instances. In the movement from the virtual Idea green to the actual expression of this Idea in a "green apple," the virtual excess of the Idea green is reduced to only a small number of its actual instances. Deleuze describes this reduction as the displacement and disguise of the individuating difference.

It remains a bit unclear how the asymmetrical synthesis between the virtual and the actual comes into being. To explain this, Deleuze introduces three important notions that are strongly related to each other: repetition, implication and explication. According to Deleuze, the intensive drama must be understood as the interplay between two kinds of repetition: a material and a hidden repetition. The difference between these repetitions is explained in terms of implication and explication. Implication concerns the intensive drama; explication concerns the actual concept in which the virtual Idea is embodied through the intensive drama.

The virtual Idea green is only explicated and unfolded in implicating the Ideas yellow and blue. It therefore repeats yellow and blue. At the same time, green itself will be implicated by other Ideas in which it is repeated (e.g. cyan, that can be defined as a differential relation between green and blue). This means that green is both an implicating series and an implicated series. It is an implicating series when it is actualized by yellow and blue; it is an implicated series when it helps actualizing cyan. The result of this is "that it returns to itself as many times as it returns to another. ${ }^{26}$ As implicating series, green returns to itself. As such, it is the ground for its own material repetition. It is thus, within the implicating series that green becomes explicated and unfolded. However, this explication is made possible, not by green itself, but by the series it implicates (variations of yellow and blue as the intensive dramas of green). As implicated series, green returns to other series and becomes the ground for its hidden repetition in other series. Thus green is both an intensive drama (as implicating series) and a virtual Idea (as implicated series).

The implicated multiplicity of the intensive drama, incarnating the virtual Idea green in actual circumstances:

26 Ibid., 300. 
- The actually undetermined. The excess of the virtual Idea green that has the potential to become actually determined as green through an asymmetrical synthesis (the range of variations implied by yellow/blue).

- The actually determinable. The conditions under which something is actually determinable as green: whenever the differentials yellow and blue actually enter into a differential relation and become the 'implicated series of green (individuation of yellow/blue).

- The actual determination. The emergence of the implicating series green in which the excess of the virtual Idea green becomes more and more reduced through its explication in actual circumstances (actual values of yellow/ blue).

This is why Deleuze agrees with Leibniz that the Cartesian criterion of clearand-distinct perceptions should be divided in two separate dimensions: the distinct-but-obscure of the virtual Idea and the clear-but-confused of the intensive drama. This can again be explained with the help of my example of the color green. As we saw, green is the result of a differential relation between the 'implicated series yellow and blue. As such, both series are distinct-butobscure. They are distinct because the color green cannot exist without the differential relation between them; they are obscure because neither yellow nor blue is drawn into clarity, both colors are only perceived unconsciously. The implicating series of the color green, on the other hand, is clear-butconfused. It is clear because the differential relation that defines green is drawn into clarity in such a way that a clear perception of the color green is established; it remains confused, because it expresses its constituting colors (yellow and blue) only in a confused way. The example of the color green makes clear that all the intensive dramas are implicated by one another "such that each continues to express the changing totality of Ideas, the variable ensemble of differential relations." 27 To get a grip on this transition from the Idea green to the "changing totality of all Ideas," I will now briefly turn to the logic of expression that Deleuze derives from Spinoza.

Deleuze's reading of Spinoza is a creative response to the problems that animate Spinoza's thinking, rather than an interpretation in the traditional sense. It would be wrong, therefore, to dismiss Expressionism in Philosophy: Spinoza 
as just another book on the history of philosophy. Rather, I would suggest that Deleuze derives from his reading of Spinoza a very rigorous and rich framework on which he builds the system of heterogenesis developed in Difference and Repetition and subsequent works. This framework is defined by what Deleuze calls the logic of expression. It is with the help of this framework that the transition from "the single Idea green" to "the changing totality of virtual Ideas" can be explained.

Two aspects of the logic of expression are of particular importance for understanding Deleuze's conception of plurality. First, expression takes place on three levels at once. As Deleuze writes, "[e]xpression presents us with a triad. In it we must distinguish substance, attributes and essence. Substance expresses itself, attributes are expressions, and essence is expressed." 28 In Spinoza's vocabulary, this first movement of expression is called natura naturans. Second, Spinoza introduces a second movement of expression. Substance first expresses itself within itself. After that, it re-expresses itself in its effects. This second movement is called natura naturata.

Deleuze shows that Spinoza has come a long way in developing a conception of plurality that is based on difference. However, in Spinoza there is still a principle of identity at work. In the interplay between substance and its effects (modes), priority is given to substance as the immanent cause of all its effects. In other words, the effects are dependent on substance, but substance is not dependent on its effects. Deleuze wants to get rid of this order of priority. To his mind, the dependence must go in both directions. The effects must be dependent on their immanent cause and the immanent cause must be dependent on its effects. That is why he proposes to "to make substance turn around the modes [the effects]-in other words, to realize univocity in the form of repetition in the eternal return." 29 This implies that it is no longer possible to make a distinction between a first and a second movement of expression. Rather, expression must be conceived as a single movement that contains within itself its own re-expression.

Deleuze's system of heterogenesis can be defined in terms of the threefold logic of expression that Deleuze finds in Spinoza. For Deleuze, to exist means one of three things: (1) to be the expresser, (2) to be the expression or (3) to be the expressed. Thus, existence has three levels of expression. Outside of these levels, nothing exists. This is of course a very schematic and simplified picture of the logic of expression. Nevertheless, it makes it possible to explain the relation between intensive dramas, actual concepts and virtual Ideas. This can be 
made clear with the help of the Japanese art of folding an origami paper. ${ }^{30}$ In origami, complex figures are produced by folding and refolding a single piece of paper (corresponding to the univocity of being). This single piece of paper is not cut into pieces, nor is anything added to it. For this reason, the origami paper can be used as an image of univocal being that expresses itself. Thus the three components of expression can be reformulated in terms of the origami paper: (1) the single piece of paper that expresses itself; (2) the folded figures that express something else, namely (3) the virtual Ideas that are expressed by them. Imagine, for instance, that the paper is folded in the form of two human figures: Peter and Paul. It is the paper that expresses itself by being folded in certain figures. Each of these figures, in their turn, expresses an Idea. One figure expresses the Idea Peter, the other the Idea Paul.

This image of the origami paper can give us an estimation of Deleuze's conception of plurality. For that to happen, the single piece of paper-understood as univocity of being-should first be re-interpreted in terms of difference. Instead of a single piece of paper that is still unfolded, we should presuppose an infinite collection of differences that is constantly folding and refolding itself. This infinite collection of differences is itself the result of previous folds and refolds. Thus, it is not the unity of the piece of paper that expresses itself, but rather its difference. This results in a peculiar type of univocity that Deleuze characterizes as follows: "Being is said in a single and same sense of everything of which it is said, but that of which it is said differs: it is said of difference itself." 31 The paper is, so to say, made out of differences; differences that are not determined from outside, but that are different in themselves.

According to Deleuze, the logic of expression is constituted by two movements: implication and explication. "Expression in general involves and implicates what it expresses, while also explicating and evolving it." 32 In the process of its unfolding, the infinite collection of differences explicates and evolves itself. At the same time, it is involved in this unfolding in two ways: as an implicating series that is clear-but-confused and as an implicated series that is distinct-but-obscure. It is here that we find the re-expression that Spinoza was talking about. The two movements of expression, explication and implication, are not opposed to each other, but they are part of one and the same process. Deleuze calls this process complication. Complication indicates both the presence of the infinite collection of differences in the Ideas that 
are expressed by it and the presence of the Ideas in the infinite collection of differences that expresses them.

Against this background it becomes possible to understand a rather strange example that Deleuze finds in a footnote of Spinoza's Short Treatise. ${ }^{33}$ In Deleuze's account, Spinoza tells us the following:

...picture a white wall. A wall that is completely white. There is nothing on it. Then you approach it with a pencil, you create a figure and then next to it you draw another. Look, your two figures exist. They exist insofar as what? They exist insofar as you have traced them. There are two characters on the white wall. You can name these two characters Peter and Paul. Would it be possible that, as long as nothing has been traced on the white wall, something else exists that is distinct from it? ${ }^{34}$

In the Short Treatise, Spinoza seems to suggest that this is not possible. If there are no figures traced on the white wall, it is impossible to distinguish something on it. However, Deleuze refuses to accept this suggestion. In his view, Spinoza's example poses a problem. Instead of solving this problem, Spinoza uses it as a practical exercise in thinking, which forces the reader to solve the problem himself. How should we formulate this problem? It is a problem of distinction. Can there be a distinction between existing things that is no longer extrinsic, but rather intrinsic? Deleuze thinks that this is indeed possible. According to him, we can make a distinction between the various degrees of intensity of the white wall. These degrees of intensity are distinct from each other, even though they cannot be separated from the white wall that includes them. The degrees of intensity of the color white "are not added to whiteness as one thing to another thing, like a shape added to the wall on which it is drawn; its degrees of intensity are intrinsic determinations, intrinsic modes, of a whiteness that remains univocally the same under whichever modality it is considered." 35 In other words, the difference in intensity is a difference

33 Benedictus de Spinoza, Short Treatise, in The Collected Works of Spinoza, ed. Michael L. Morgan (Indianapolis: Hackett Publishing Company, 2002), 91 (note 21).

34 Gilles Deleuze, “10-03-81: Infini actuel-éternité” (my translation). Between December 1980 and March 1981, Deleuze gave a series of fourteen seminars on Spinoza at the Université de Paris VIII-Vincennes à St. Denis Gilles. They are available online at www.webdeleuze. com/php/sommaire.html. See also Deleuze's commentary in Deleuze, Expressionism in Philosophy, 195-6.

35 Ibid., 196. Deleuze derives his understanding of intensity from Duns Scotus. For more on this, see Daniel W. Smith, Essays on Deleuze (Edinburgh: Edinburgh University Press, 2012), 28-37. See also Duffy, The Logic of Expression. 
of the white within the white itself. It is an intrinsic difference. Thus Deleuze gives us another way of understanding intensive dramas.

For Deleuze, it is important that "the shape on the white wall" and "the degree of intensity within the white wall" are both understood as physical realities. The degree of intensity can be said to have a physical reality because it has a certain quantity. Not an extensive quantity that is determined from outside (like the shapes that are drawn on the wall), but an intensive quantity that has an intrinsic determination and is something in itself. According to Deleuze, the whiteness of the wall can thus be perceived from two perspectives. If we look at the white wall as such, when there are no shapes drawn on it, the whiteness must be taken as an undivided continuum of degrees in intensity. Moreover, each degree of intensity agrees with all the others. From this perspective, the white wall corresponds to the changing totality of virtual Ideas. However, if we look at the white wall after certain shapes have been drawn on it, the continuum of the whiteness has been divided in intensive quantities, each of which is limited by all the others. These intensive quantities can only be determined in relation to the shapes that are drawn on the wall.

In the case of our example, Peter differs from Paul in the sense that each of them has its own intensive quantity. The degrees of intensity belonging to Paul are different from the degrees of intensity belonging to Peter. Although these intensive quantities can only be determined in relation to the shapes on the wall, it is not the shape that defines the essence of Peter. On the contrary, the intensive quantity is the essence; the shape is only the expression of this essence in a particular concept. As a quantity, the degree of intensity of Peter has certain limits. These limits should not be understood as the outlines of an extensive shape, but as limits of actions. (Just like the edge of a forest emerges when the forest reaches the limit of its range of action). The essence of Peter, as an intensive quantity, determines what Peter can do. (Just as yellow and blue define the limits of what the color green can do). In Deleuze's reading, the essence is no longer understood in traditional terms. It is neither an original trait that defines the identity of a thing ("man is a rational animal"), nor a potential that has to be actualized ("the essence of the acorn is: becoming a tree”). Instead, the essence of a thing is defined as a differential relation that belongs to a virtual Idea. (Just as the essence of green can be defined as the differential relation between yellow and blue). Insofar as the essence of Peter (as an intensive quantity) is implicating and enveloping all the other intensive quantities (Paul, the dog, the chair, etc.), rather than being implicated and enveloped by them, it is an individuating difference. This individuating difference expresses Peter in a clear way; whereas the other things are only expressed in a confused way. However, insofar as the essence of Peter is 
implicated and enveloped by all the others rather than implicating and enveloping them, it is an individual difference. In that case, Peter is expressed in a confused way by all the others.

\section{Conclusion: The Logic of Difference and Repetition}

I have shown in this article that Deleuze's system of heterogenesis can be described in terms of the relations between actual concepts, intensive dramas and virtual Ideas. I have particularly focused on Deleuze's conception of plurality, understood in terms of differential and implied multiplicities. In his book on Spinoza, Deleuze explained the relation between these multiplicities in terms of expression; in Difference and Repetition he uses the notions difference and repetition. In this concluding section, I will show how the logic of expression is related to the dynamic of difference and repetition. According to Deleuze, there are three kinds of repetitions. (1) The material repetition of the Same, understood as "that which differs." 36 This repetition is brought about by the implicating series. (2) The hidden repetition of the Similar, understood as "that which makes dissimilar." 37 This repetition is made possible by the implicated series. (3) The eternal repetition of Difference, understood as that "which 'makes' the difference." 38 This repetition concerns the changing totality of virtual Ideas.

At first sight, one might be tempted to identify this triad with the triad of expression. However, that would be a mistake. The translation of the logic of expression to the dynamic of difference and repetition is more complex than that. As the reader might recall, the triad of expression already proved to be a set of four instead of three. This was because the expresser was re-interpreted in terms of an interplay between the implicating and the implicated series. In Diffference and Repetition we also encounter four terms: the grounded, the foundation, the ground and the unground. It is here that we can relate the logic of expression to that of difference and repetition.

This is not the place to work this out in detail. I will only be able to make some brief remarks on the relation between the three repetitions and especially on the notion of the unground. In the two repetitions that we have already discussed in detail-the material repetition of the implicating series (the foundation) and the hidden repetition of the implicated series (the ground)the difference is not given in its excessive differential potential, but is it

Deleuze, Difference and Repetition, 301.

Ibid.

Ibid., 292. 
always already reduced to some degree. Therefore a third repetition is needed that gives the virtual Idea in its excess by "making" the difference again and again. Deleuze calls this third repetition the unground.

In the 'implicating series of the first repetition, difference is disguised and displaced. This implies a movement from the excess of the Idea in which its differential potential is still infinite, to the reduction of this excess in its individuation. In the end, this results in an explication in which difference "is turned inside out and distributed in such a way as to be dispelled, compensated, equalized and suppressed in the extensity which it creates." ${ }^{39}$ In this way difference is cancelled. Not as such, but outside itself, in its own explication. In the implicated series that is hidden beneath the implicating one, difference is constantly diverged and decentered. This second repetition is the cause and the ground of the first one (under which it is buried). Deleuze indicates, however, that "it is not enough to oppose two repetitions." ${ }^{40} \mathrm{~A}$ third repetition is needed to avoid that the hidden repetition of the implicated series remains buried under the material repetition of the implicating series. Beyond the material repetition "from which difference is drawn" and the hidden repetition "which includes it," Deleuze sees "a repetition which "makes" the difference." 41 In this way the diverging and decentering movement of difference is freed from the disguise and displacement under which it was buried. Thus, through the third repetition the foundation of the first repetition and the ground of the second are annulled by an unground. This notion of the unground can only be understood in relation to Deleuze's theory of time. Here I can only give a first estimation of it with the help of the image of the dice throw that Deleuze borrows from Nietzsche and turns to his own advantage.

The image of the dice throw highlights the difference between the conception of plurality that is implied by the model of representation and the one that Deleuze tries to formulate. In the model of representation plurality is understood as sedentary distribution of being. In Deleuze's view, this sedentary distribution results in a bad game that does not take the dice throw seriously enough. This game presupposes "pre-existing categorical rules" that "serve to determine the probabilities-in other words, the winning and losing 'hypotheses." 42 The bad game does not affirm chance, but tries to exorcise it:

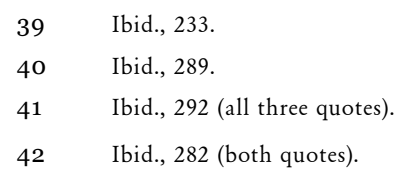


...as such, it subsumes opposing hypotheses by establishing a corresponding series of numerically distinct turns or throws which are supposed to effect a distribution among them; the outcomes or results of these throws are distributed according to their consequences following a hypothetical necessity-in other words, according to the hypothesis carried out." 43

As we saw already, the sedentary distribution privileges the identity of the hypothesis over the difference of chance and therefore it can neither account for genuine novelty nor explain real variation.

As an alternative to the sedentary distribution in which the bad game results, Deleuze introduces a conception of plurality that implies a nomadic distribution of being. This nomadic distribution is made possible by a "divine game" that includes its own regulations and is not based on pre-existing categorical rules. "As a result, every time, the whole of chance is affirmed in a necessarily winning throw" that embraces "all possible combinations and rules in the system of its own return." 44 The dice that are used in the "divine game" have an unlimited potential for variation. Moreover, during the game there will constantly emerge new sets of dice. This means that the dice-i.e. virtual Ideas-are both the cause and the effect of the throw. As cause they determine the outcome of the throw, as effect they emerge within it as a renewed set of dice, implying new rules and new combinations. Each dice will imply an excess that remains virtual (just as the differential relation between yellow and blue implies a virtual excess of green).

This has several implications. First, the actual outcome of the throw (actual concepts) will imply a virtual excess that is not known (virtual Ideas). In other words, the outcomes of the next throw cannot be predicted. Second, a new throw will be determined by the dice of all the previous ones (the totality of Ideas; the ensemble of differential relations), but it will also bring about new series of dice that will be added to all the previous ones. In other words, the throw will create new Ideas that were not part of any of the previous games. Moreover, it will bring about new configurations of already existing Ideas (the totality of Ideas changes; the ensemble of differential relations varies). Third, the divine game is simultaneously played on two tables at once (that together constitute the intensive dramas). One table shows a material repetition that disguises and displaces the difference on which it relies; the other displays a hidden repetition that diverges and decenters the difference that

43 Ibid.

44 Ibid., 283, 116 . 
it constitutes. On the first table, the dice of the color green manifest itself as a particular shade of green. In this sense, it disguises and displaces the differential relation of yellow and blue that constitutes it. On the second table, however, the dice of green manifest itself as constituent of cyan. In that sense, it diverges and decenters the differential relation that constitutes cyan. Fourth, the throw itself is repeated. This new throw, destroys the actual outcome of earlier throws, but not the set of dice (the Ideas) that made these earlier throws possible. Although this set will be changed by each throw, it will not disappear. However, the new throw is not external to the game, but it constitutes the game as such. "The different throws can then no longer be said to be numerically distinct: each necessarily winning throw entails the reproduction of the act of throwing under another rule which still draws all its consequences from among the consequences of the preceding throw." ${ }^{5}$ In other words, Deleuze conceives the world and its history in terms of a single throw that is ontologically unique and remains the same for all games, while the outcomes of these games "implicate, displace and recover their combinations in one another throughout the unique and open space of the univocal." 46 This is how the logic of expression can be translated in a logic of difference and repetition, which constitutes Deleuze's system of heterogenesis. 